\title{
Investigación en Responsabilidad Social Empresaria y Sustentabilidad Corporativa: evolución, tensiones actuales y perspectivas futuras*
}

\author{
Research on Corporate Social Responsibility and Corporate Sustainability: Evolution, current tensions, and future \\ perspectives
}

Pesquisa em Responsabilidade Social Empresarial e Sustentabilidade Corporativa: evolução, tensões atuais e perspectivas futuras

\author{
Celina N. Amato ${ }^{a}$ \\ Facultad de ciencias Económicas, Universidad Nacional de \\ Córdoba, CONICET, Argentina \\ amatocelina@eco.uncor.edu \\ ORCID: https://orcid.org/0000-0002-9136-0516
}

DOI: https://doi.org/10.11144/Javeriana.cao34.irsesc

\section{Resumen:}

El artículo tiene como objetivo comprender la evolución de los constructos Responsabilidad Social Empresaria y Sustentabilidad Corporativa de acuerdo con distintos paradigmas de la investigación en management, así como también las tensiones actuales y posibilidades de investigación futura. La metodología elegida es la revisión terciaria de literatura. Como resultados se destacan la situación de convergencia de ambos conceptos, que dificulta la claridad para el avance de la investigación y las críticas hacia ellos (business case, profecía autocumplida, invisibilización de stakeholders y reproducción del paradigma capitalista de producción y consumo). Las perspectivas futuras de investigación se relacionan con la profundización de cada constructo por separado, la incorporación de otras teorías y disciplinas al análisis y la integración del pensamiento de corrientes críticas.

Códigos JEL: M14, Q01, Q56

Palabras clave: Estudios críticos en administración, revisión terciaria de literatura, triple cuenta de resultados.

\section{Abstract:}

The paper aims to understand the evolution of the Corporate Social Responsibility and Corporate Sustainability constructs according to different management research paradigms, as well as current tensions and future research possibilities. The methodology adopted is a tertiary review of the literature. The situation of the convergence of the two concepts, which makes it difficult to have clarity for the advance of research, and criticism towards both constructs (business case, self-fulfilling prophecy, invisibility of stakeholders, and reproduction of the capitalist paradigm of production and consumption), stand out as results. Future research perspectives are related to the deepening of each construct separately, the incorporation of other theories and disciplines into the analysis, and the integration of nonmainstream ideas.

JEL Codes: M14, Q01, Q56

Keywords: Critical management studies, tertiary literature review, triple bottom line.

\section{Resumo:}

O artigo visa entender a evolução dos conceitos Responsabilidade Social Corporativa e Sustentabilidade Corporativa, segundo distintos paradigmas de pesquisa em managment, e as tensões atuais e as possibilidades de pesquisas futuras. A metodologia utilizada é a revisão terciária de literatura. Nos resultados, se destaca a situação de convergência de ambos os conceitos o qual dificulta a clareza para o avance nas pesquisas e as críticas em relação a eles (bussines case, profecia auto-cumprida, encobrimento de stakeholders e reprodução do paradigma capitalista de produção e consumo). As perspectivas futuras de pesquisa se relacionam com o estudo a fundo de cada conceito por aparte, a incorporação de outras teorias e disciplinas na análise e a integração do pensamento de correntes críticas.

Códigos JEL: M14, Q01, Q56

Palavras-chave: Estudos críticos em administração, revisão terciária de literatura, triplo resultado final.

Notas de autor

a Autora de correspondencia. Correo electrónico: amatocelina@eco.uncor.edu 


\section{Introducción}

En la literatura del management hay bastantes escritos acerca de la relación entre empresa, ambiente y sociedad, en los cuales los autores utilizan diversos conceptos para referirse a ello. Dos de los constructos más utilizados son Responsabilidad Social Empresaria -RSE- y Sustentabilidad Corporativa -SC-, precisamente, es menester de este trabajo analizar como ha sido abordado cada uno de ellos desde la investigación.

El origen de la RSE puede encontrarse en el famoso trabajo de Bowen (1953) acerca de la moralidad de los empresarios -incluso mucho antes si pensamos en Carnegie y la filantropía o en las funciones morales de los ejecutivos de Barnard- bajo un paradigma del management asociado a la moralidad, la obligación y el deber social (Carroll, 1999; Drucker, 1954; Acquier, Gond \& Pasquero, 2011).

El origen de la SC se asocia a los límites demostrados por los recursos naturales dado el crecimiento económico ilimitado (Meadows, Meadows, Randers \& Behrens, 1972). Ya en 1960 libros como La Primavera Silenciosa de Carson y La Bomba Demográfica de Ehrlich planteaban preguntas con respecto a estos límites (Latapí Agudelo, Jóhannsdóttir \& Davídsdóttir, 2019). Posteriormente, el aumento del interés por la SC puede ser atribuido al crecimiento de los grandes problemas globales y la percepción social de que las empresas debían proveer soluciones a ellos (Meuer, Koelbel \& Hoffmann, 2020). Esta situación se pone de manifiesto cuando adquiere popularidad el término desarrollo sostenible -DS- (Comisión Mundial sobre Medio Ambiente y Desarrollo - CMMAyD, 1987); aunque teóricamente son conceptos diferentes (Castro, 2004; Gladwin, Kennelly \& Krause, 1995; Lélé, 1991). Esta visión se asocia a un nuevo paradigma de inclusión del debate "verde" en Administración (Linnenluecke \& Griffiths, 2013).

La literatura ha encontrado abordajes diferenciados para estos constructos lo cual permite pensar que estamos ante enfoques distintos, con raíces teóricas diferentes (Amato, Buraschi \& Peretti, 2016; Ashrafi, Adams, Walker \& Magnan, 2018; Bansal \& Song, 2017; Meuer et al., 2020; Montiel, 2008). Pero en la actualidad ambas conceptualizaciones incluyen la integración de las dimensiones económicas, sociales y ambientales (Montiel, 2008) o de la triple cuenta de resultados -TBL por sus siglas en inglés- (Elkington, 1994, 1997, 2004) y muchas de las definiciones y construcciones teóricas son convergentes (Bansal, 2005; Carroll, 1999; Montiel, 2008; Montiel \& Delgado-Ceballos, 2014).

Esta situación ha propiciado la existencia de un campo difuso que ha frenado el crecimiento en la investigación (Bansal \& Song, 2017), dada la importancia de tener bien definidos, claros y acordados los constructos para poder reproducir resultados (Meuer et al., 2020; Montiel, 2008). Esta convergencia ha dado lugar a la aparición de críticas hacia ambos términos que constituyen tensiones importantes a tener en cuenta para el avance de la investigación.

En este contexto se plantean los siguientes interrogantes: ¿Cuál ha sido la evolución de la investigación en ambos constructos? ¿Cuáles son sus diferencias y similitudes? ¿Cuáles son las tensiones actuales? y ¿Qué perspectivas a futuro tienen?, siendo el objetivo de este artículo analizar la evolución y estado del arte de la investigación en RSE y SC con el fin de detectar diferencias y similitudes, y ensayar posibles perspectivas de investigación futura en función de las tensiones actuales.

El artículo plantea una revisión orientada teniendo en cuenta aspectos diferenciales. Se trata de una revisión terciaria de literatura con énfasis en la selección de artículos con mayor impacto, lo cual no ha sido contemplado con anterioridad. Se profundiza en la separación de ambos constructos, tal como lo reclama la literatura reciente (Ashrafi et al., 2018; Bansal \& Song, 2017), continuando la investigación en un tópico tan crucial para la academia y la sociedad como lo es la RSE y la SC (Etzion, 2007). Se incorporaron artículos de journals que no responden al mainstream (específicamente los estudios críticos en administración-ECA- ${ }^{1}$ ), considerándose también distintivo el estar escrito en español y dirigido a la comunidad hispano-hablante (Sanabria, Saavedra, \& Smida, 2015). 
El artículo se estructura de la siguiente manera: a continuación se explicita la metodología; en la discusión se desarrolla la evolución de la investigación de ambos conceptos y sus principales diferencias, similitudes, tensiones actuales y perspectivas a futuro; finalmente, se incluyen algunas conclusiones y limitaciones.

\section{Metodología}

La metodología elegida fue la revisión bibliográfica orientada que se llevó a cabo en dos etapas. En la primer etapa, el objetivo fue encontrar artículos de revisiones de literatura previas (e.g. Martins \& Pato, 2019) sobre los constructos RSE y SC, sin fines de exhaustividad ni de sistematización bibliométrica sino para profundizar el análisis de acuerdo con el objetivo planteado. Dados los criterios de búsqueda y la selección arbitraria, quedaron afuera aquellos reviews que relacionan estos temas con otros importantes (como economía circular, transición a la sustentabilidad, desarrollo sustentable, políticas públicas sustentables, etc.).

La elección de estas revisiones se realizó teniendo en cuenta criterios de calidad, como la búsqueda a través de bases de datos $-\mathrm{BD}$ - reconocidas y el número de citas de los artículos. Se utilizaron determinados algoritmos de búsqueda y se seleccionaron las publicaciones que aparecieron dentro de las primeras veinte en cada $\mathrm{BD}$, ordenadas de acuerdo con el número de citas o relevancia, y que estuvieran estrechamente relacionadas con el objetivo de este trabajo. Además, se incluyó un algoritmo específico para incorporar en los resultados artículos de corrientes críticas. Esta etapa se resume en tabla 1.

TABLA 1

Criterios de búsqueda para la elección de artículos de la etapa 1 de la metodología

\begin{tabular}{|c|c|c|c|c|}
\hline Base & $\begin{array}{c}\text { A goritmos (en } \\
\text { título o pa abbras } \\
\text { clave) }\end{array}$ & Área/especialidad & Orden & Recurso \\
\hline Scopus & \multirow{5}{*}{$\begin{array}{l}\text { - Corporate } \\
\text { social } \\
\text { responsibility } \\
\text { - Sustainability } \\
\text { - Review }\end{array}$} & $\begin{array}{l}\text { - Social science } \\
\text { - Business, Management and } \\
\text { Accounting } \\
\text { - Economics, Econometrics } \\
\text { and Finance }\end{array}$ & Mayores citas & Journal \\
\hline $\begin{array}{l}\text { Emerald } \\
\text { Insight }\end{array}$ & & (Filtros no disponibles) & Relevancia & Article \\
\hline Jstor & & $\begin{array}{l}\text { - Development Studies } \\
\text { - Management } \\
\text { Organizational Behavior }\end{array}$ & $\begin{array}{l}\text { (Orden no } \\
\text { disponible) }\end{array}$ & Articles \\
\hline $\begin{array}{c}\text { Ebsco } \\
\text { Host }\end{array}$ & & $\begin{array}{l}\text { - Social responsibility of } \\
\text { business } \\
\text { - Sustainable development } \\
\text { - Sustainability } \\
\text { - Business ethics } \\
\text { - Social responsibility } \\
\text { - Literature reviews }\end{array}$ & Relevancia & $\begin{array}{l}\text { Publicaciones } \\
\text { académicas } \\
\text { (arbitradas) }\end{array}$ \\
\hline $\begin{array}{l}\text { Science } \\
\text { Direct }\end{array}$ & & - Review articles & Relevancia & $\begin{array}{l}\text { Review } \\
\text { articles }\end{array}$ \\
\hline $\begin{array}{l}\text { Todas } \\
\text { las BD }\end{array}$ & Critical & (Especificados de cada base) & $\begin{array}{c}\text { (Especificados } \\
\text { de cada base) }\end{array}$ & $\begin{array}{l}\text { (Especificados } \\
\text { de cada base) }\end{array}$ \\
\hline
\end{tabular}

Fuente: elaboración propia.

De acuerdo con estos criterios, el total de reviews seleccionados fueron 29 y se resumen en tabla 2. En la tablaA1 del anexo se detallan sus principales características. 
TABLA 2

Reviews seleccionadas en la etapa 1 de la metodología (ordenados cronológicamente)

\begin{tabular}{|c|c|c|c|}
\hline$\#$ & Autores & Año & Citas* \\
\hline 1 & Lélé & 1991 & 3.204 \\
\hline 2 & Elkington** & 1994 & 3.584 \\
\hline 3 & Gladwin, Kennelly, \& Krause & 1995 & 2.819 \\
\hline 4 & Elkington** & 1997 & 120 \\
\hline 5 & Mebratu & 1998 & 1.668 \\
\hline 6 & Carroll & 1999 & 10.446 \\
\hline 7 & Moir & 2001 & 1.412 \\
\hline 8 & Garriga \& Melé & 2004 & 5.612 \\
\hline 9 & Elkington** & 2004 & 539 \\
\hline 10 & McWilliams, Siegel, \& Wright & 2006 & 3.722 \\
\hline 11 & Glavič \& Lukman & 2007 & 1.050 \\
\hline 12 & Dahlsrud & 2008 & 5.034 \\
\hline 13 & Lee & 2008 & 1.873 \\
\hline 14 & Montiel & 2008 & 771 \\
\hline 15 & Lozano & 2008 & 729 \\
\hline 16 & Carroll \& Shabana & 2010 & 4.118 \\
\hline 17 & Scherer \& Palazzo & 2011 & 1.895 \\
\hline 18 & Aguinis \& Glavas & 2012 & 3.000 \\
\hline 19 & Lang, Wiek, Bergmann, et al. & 2012 & 1.742 \\
\hline 20 & Brandt, Ernst, Gralla, et al. & 2013 & 563 \\
\hline 21 & Montiel \& Delgado-Ceballos & 2014 & 378 \\
\hline 22 & Bansal \& DesJardine & 2014 & 403 \\
\hline 23 & Lozano, Carpenter, \& Huisingh & 2015 & 205 \\
\hline 24 & Hahn, Pinkse, Preuss, \& Figge & 2015 & 494 \\
\hline 25 & Bansal \& Song & 2017 & 237 \\
\hline 26 & Ashrafi, Adams, Walker, \& Magnan & 2018 & 38 \\
\hline 27 & Olawumi \& Chan & 2018 & 180 \\
\hline 28 & Latapí Agudelo Jóhannsdóttir, \& Davídsdóttir & 2019 & 70 \\
\hline 29 & Meuer, Koelbel, \& Hoffmann & 2020 & 11 \\
\hline
\end{tabular}

* Se recurrió a una BD general para el número de citas, tomando Google Scholar (https://scholar.google.com) al 28/10/2020.

** En Elkington no se tuvieron en cuenta las citas, dado que es el creador de la TBL y sus trabajos aportan en esta creación. Fuente: elaboración propia.

En la segunda etapa se prosiguió con la revisión bibliográfica hacia delante y hacia atrás de los artículos de tabla 2, con el fin mejorar la representación de artículos relevantes. Para este proceso el criterio fue escoger publicaciones que estuvieran citadas por al menos cuatro de los artículos de la tabla 2. Así, surgieron otros 30 trabajos que se resumen en tabla 3. 
TABLA 3

Artículos seleccionados en la etapa 2 de la metodología (ordenados por frecuencia)

\begin{tabular}{|c|c|c|c|}
\hline \# & Autores & Año & Frecuencia \\
\hline 1 & Comisión Mundial sobre Medio Ambiente y Desarrollo & 1987 & 18 \\
\hline 2 & Freeman & 1984 & 11 \\
\hline 3 & Bowen & 1953 & 9 \\
\hline 4 & Frederick & 1960 & 9 \\
\hline 5 & Orlitzky, Schmidt \& Rynes & 2003 & 9 \\
\hline 6 & Friedman & 1970 & 8 \\
\hline 7 & Shrivastava & 1995 & 8 \\
\hline 8 & Wood & 1991 & 8 \\
\hline 9 & Bansal & 2005 & 7 \\
\hline 10 & Davis & 1973 & 7 \\
\hline 11 & Donaldson \& Preston & 1995 & 7 \\
\hline 12 & Friedman & 1962 & 7 \\
\hline 13 & Hart & 1995 & 7 \\
\hline 14 & Margolis \& Walsh & 2003 & 6 \\
\hline 15 & Porter \& Kramer & 2006 & 6 \\
\hline 16 & Ackerman & 1973 & 5 \\
\hline 17 & Clarkson & 1995 & 5 \\
\hline 18 & Hoffman & 1999 & 5 \\
\hline 19 & Meadows, Meadows, Randers \& Behrens & 1972 & 5 \\
\hline 20 & Swanson & 1995 & 5 \\
\hline 21 & A guilera, Rupp, Williams \& Ganapathi & 2007 & 4 \\
\hline 22 & Bamard & 1938 & 4 \\
\hline 23 & Doh \& Guay & 2006 & 4 \\
\hline 24 & Jones & 1980 & 4 \\
\hline 25 & Preston \& Post & 1975 & 4 \\
\hline 26 & Russo \& Fouts & 1997 & 4 \\
\hline 27 & Banerjee* & 2008 & 3 \\
\hline 28 & Drucker* & 1954 & 3 \\
\hline 29 & Etzion* & 2007 & 3 \\
\hline 30 & Valente* & 2012 & 3 \\
\hline
\end{tabular}

* Estos trabajos se incluyen excepcionalmente porque sus autores tenían mucha frecuencia con otros artículos, aunque no llegaran al mínimo de cuatro con la obra aquí referida.

Fuente: elaboración propia.

Finalmente, el conjunto total de artículos seleccionados para el análisis fue de 59. Por cuestiones de extensión no se incluyen aquí aspectos descriptivos de este conjunto, pero pueden ser solicitados a la autora por correo electrónico.

\section{Discusión}

\section{Evolución histórica}

La evolución histórica de la investigación en management sobre ambos constructos se analiza considerando cuatro etapas asociadas a distintos paradigmas ${ }^{2}$, en las cuales se destacan las distinciones teóricas entre RSE y SC, sus similitudes, y las tensiones actuales; todo lo cual se sintetiza en la figura 1. 


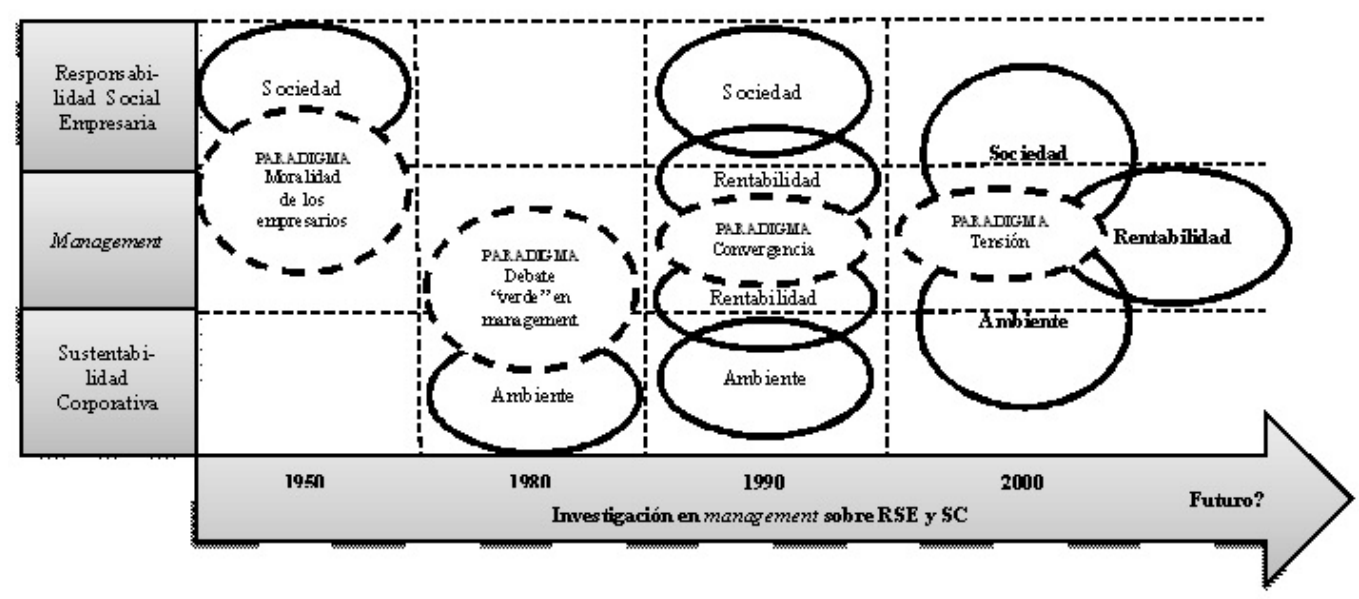

FIGURA 1

Evolución histórica de la investigación en RSE y SC

Nota: el paradigma de la tensión recurre al clásico diagrama de Venn sin intensiones de seguir tradiciones científicas reduccionistas y conociendo que existen alternativas más completas (e.g. Asrafi et al., 2018; Lozano, 2008). Fuente: elaboración propia en base a Bansal y Song (2017).

En el periodo de tiempo analizado se identifican claramente cuatro paradigmas: 1) moralidad de los empresarios, asociado al business ethics y luego al business \& society; 2) límites del crecimiento o la inclusión del debate "verde" en Administración; 3) convergencia de la rentabilidad con la sociedad y el ambiente o de la RSE y la SC; y 4) tensiones en la relación empresa-sociedad-ambiente. La idea de “¿Futuro?” de la figura 1 es el posible inicio de un quinto paradigma ${ }^{3}$, en el cual la comunidad científica tiene conocimiento de los anteriores y se pregunta ¿cómo avanzar con estas tensiones?, en un contexto en el cual las firmas ya no pueden eludir estos temas sino que deben ser parte de sus estrategias y decisiones (Latapí Agudelo et al., 2019).

Estos paradigmas se desarrollan a continuación.

\section{Distinciones teóricas: moralidad de los empresarios y debate "verde" en Administración}

Entre 1950 y 1960 comienza la investigación en RSE cuestionando los límites del capitalismo, con base en el razonamiento normativo y la economía del bienestar para juzgar la idoneidad de las acciones de las empresas (para una descripción detallada de los orígenes de la RSE, anteriores a 1950, ver Latapí Agudelo et al., 2019). En management argumentaron que las responsabilidades morales hacia la sociedad y los marcos legales deberían guiar las decisiones de los gerentes (Bansal \& Song, 2017). Expresaron preocupación por las cuestiones sociales siendo el marco teórico inicial el del valor del accionista o shareholder en contraposición a los derechos de las partes interesadas o stakeholders (Bowen, 1953; Friedman, 1970). Uno de los detractores más citados al respecto es Friedman por haber asegurado que la única responsabilidad social del accionista era la de maximizar las ganancias de la empresa (Friedman, 1970).

Los trabajos de Barnard (1938), Bowen (1953) -considerado en gran parte de la literatura como el padre de la RSE (Acquier et al., 2011)-, Drucker (1954), Frederick (1960), Ackerman (1973), Davis (1973), Preston y Post (1975), aparecen como los destacados en cuanto a esta primera aproximación de la RSE (Bansal \& Song, 2017; Carroll, 1999; Latapí Agudelo et al., 2019; Lee, 2008). La dimensión ambiental no estaba incluida en las primeras definiciones (Carroll, 1999; Dahlsrud, 2008), ni en las teorías y enfoques utilizados (Garriga \& Melé, 2004).

Los trabajos iniciales se centraban en la moralidad de los empresarios, siendo el foco el business ethics y sólo hasta fines de la década de 1970 comienzan a virar la mirada hacia el business \& society (Lockett, Moon \& Visser, 2006; Perdomo \& Escobar, 2011) influenciada, en parte, por la proliferación de movimientos sociales y de legislación social y ambiental (Latapí Agudelo et al., 2019). Las empresas comienzan a centrarse en la 
implementación de la RSE más que en la discusión de si debían o no hacerlo (Lee, 2008). Se comienza a ver a las personas de negocios preocupadas por la filantropía y las relaciones con la comunidad (Carroll, 1999), y surgen las firmas más renombradas de RSE: Body Shop en el Reino Unido y Ben \& Jerry's en Estados Unidos (Latapí Agudelo et al., 2019).

Paralelamente, entre 1970 y 1980 otro grupo de investigadores comenzaron a alertar sobre los efectos perjudiciales del crecimiento económico ilimitado sobre el ambiente, dados los límites demostrados por los recursos naturales (Meadows et al., 1972). Mebratu (1998) destaca los acontecimientos que forjaron este paradigma: las obras de los economistas Malthus y Ricardo; la Conferencia sobre Ambiente Humano de la ONU en Estocolmo (1972); el reporte del Club de Roma (1972); el libro Small is beautiful de Schumacher (1979); el Programa de Ambiente de la ONU (1978); la Estrategia Mundial de Conservación (1980); y el Reporte Brundtland (CMMAyD, 1987), que popularizó el término desarrollo sostenible -DS- ${ }^{4}$.

Los investigadores del management aceptaron este nuevo paradigma y comenzaron a trabajar el concepto de SC. Linnenluecke y Griffiths (2013) llaman este proceso el debate "verde" en Administración cuando se empieza a incluir al ambiente natural como tercer pilar de responsabilidad de una empresa. El aumento del interés por la SC puede ser atribuido a la emergencia de los grandes problemas globales y la percepción social que las empresas deben proveer soluciones a ellos (Meuer et al., 2020), y crece cuando comienza a adquirir popularidad el término DS. Aunque la literatura analizada encuentra que SC y DS son conceptos claramente distintos (Ashrafi et al., 2018; Castro, 2004; Gladwin et al., 1995; Lélé, 1991; Meuer et al., 2020; Montiel, 2008; Olawumi \& Chan, 2018).

Estos investigadores se centraron en que las empresas eran sistemas anidados en otros sistemas mucho más amplios -macroeconómicos, políticos, sociales y ecológicos- siendo el centro las cuestiones ambientales. Argumentaron que el exceso de producción industrial y de consumo humano estaba contribuyendo a los desequilibrios ambientales, que en última instancia socavarían los sistemas empresariales y económicos (Gladwin et al., 1995; Hart, 1995; Meadows et al., 1972; Shrivastava, 1995). Siguiendo a Montiel y DelgadoCeballos (2014), el término SC se incorpora por primera vez cuando Gladwin et al. (1995) lo describen y se profundiza cuando Bansal (2005) lo operacionaliza como un constructo tridimensional. Meuer et al. (2020), en su análisis de definiciones seminales de SC, incluye como primeros los trabajos de Elkington (1997) y Atkinson (2000).

Como se decanta, las raíces teóricas de RSE y SC son diferentes. Bansal y Song (2017) realizan un detallado análisis sobre este aspecto indicando que los orígenes de cada uno se remiten, de alguna manera, a la distinción entre ética y ciencia. Las raíces en la ética de la RSE dan sentido a los valores detrás de la gestión empresarial, por ello que sus primeros autores hablaban de moralidad, obligación y deber social (Bowen, 1953; Carroll, 1999; Drucker, 1954) y, a través de esta comprensión ayudar a prescribir acciones. Las raíces en la ciencia de la SC se sustentan en que el impacto de las actividades empresariales en el entorno natural era tan significativo que impedía el bienestar social, pretendiendo descubrir las causas de este fenómeno y dando importancia a los hechos más que a los valores asociados a las mismas.

También existen diferencias en cuanto a las asunciones sobre para quién o para qué una empresa debía ser responsable o sostenible. La RSE considera a la empresa como un actor social (McWilliams, Siegel, \& Wright, 2006) que se encuentra en el centro de la escena y desde allí se relaciona con sus stakeholders debiendo satisfacer sus demandas (Bansal \& Song, 2017), destacándose la influencia de esa teoría (Donaldson \& Preston, 1995; Freeman, 1984). En cambio, la SC ve a las empresas como sistemas anidados dentro de otros sistemas, incluidos los sistemas sociales y naturales (Gladwin et al., 1995), donde todos están interconectados (Montiel, 2008). Los investigadores no asumen a ningún actor como central (Bansal \& Song, 2017) pero destacan la importancia de la preservación del ambiente para la subsistencia de todos los sistemas.

Por último, podemos señalar distinciones en la concepción de cada constructo sobre el ambiente natural. La RSE se relaciona con el tecnocentrismo, que refleja el dominio humano sobre la naturaleza, y el antropocentrismo en el cual hay un valor de uso del ambiente; versus la SC que se relaciona con el 
ecocentrismo en el cual existe un valor intrínseco del ambiente, independiente del valor para la humanidad (Bansal \& Song, 2017; Gladwin et al., 1995; Montiel, 2008). Y también, desde una mirada temporal: algunos autores argumentan que el concepto de SC posee una noción de largo plazo; y el de RSE, de corto plazo (Asrafi et al., 2018; Bansal \& Desjardine, 2014; Lozano, 2008).

Estas diferencias principales se resumen en tabla 4. Es de esperar que los investigadores que siguen a cada uno de estos enfoques puedan continuar explotándolos centrados en los asuntos distintivos que competen a cada cual.

TABLA 4

Principales diferencias entre los constructos de RSE y SC

\begin{tabular}{lll}
\hline \multicolumn{1}{c}{ Aspecto } & \multicolumn{1}{c}{ RSE } & \multicolumn{1}{c}{ SC } \\
\hline Primeras definiciones & $\begin{array}{l}\text { Asociadas a cuestiones } \\
\text { sociales relacionadas con la } \\
\text { moralidad, la obligación y el } \\
\text { deber social. }\end{array}$ & $\begin{array}{l}\text { Asociadas a cuestiones } \\
\text { ambientales, relacionadas con } \\
\text { la gestión ecológica y su } \\
\text { relación con el desarrollo } \\
\text { económico. }\end{array}$ \\
\hline $\begin{array}{l}\text { Para quién o para qué las } \\
\text { empresas debían ser } \\
\text { responsables o sostenibles }\end{array}$ & $\begin{array}{l}\text { Para satisfacer las demandas } \\
\text { de los stakeholders }\end{array}$ & $\begin{array}{l}\text { Para preservar el medio } \\
\text { ambiente natural }\end{array}$ \\
\hline Origen té́rico & Ética (valores) & Ciencia (hechos) \\
\hline Concepción del ambiente & $\begin{array}{l}\text { Tecnocéntrico (dominio } \\
\text { humano sobre la naturaleza) } \\
\text { Antropocéntrico (valor de uso } \\
\text { del ambiente) }\end{array}$ & $\begin{array}{l}\text { Ecocéntrico (rechazo del } \\
\text { dominio humano sobre la } \\
\text { naturaleza, pensamiento } \\
\text { sistémico, valor intrínseco del } \\
\text { ambiente) }\end{array}$ \\
\hline Noción temporal & Corto plazo & Largo plazo \\
\hline
\end{tabular}

Fuente: elaboración propia con base en Asrafi et al. (2018), Bansal y Desjardine (2014), Bansal y Song (2017); Gladwin et al. (1995), y Montiel (2008).

\section{Similitudes: convergencia}

A partir de 1990 inicia una etapa de convergencia en la investigación en la cual ambos términos comenzaron a utilizarse indistintamente. Los investigadores de RSE reconocen que las cuestiones sociales incluyen la gestión del ambiente, y los de SC consideran a la sociedad como un elemento importante en los sistemas ambientales, intercambiando también enfoques y teorías.

Los administradores comenzaron a buscar y aplicar técnicas, herramientas y modelos que los ayudaran con la intersección de los campos económico-social (e.g. Swanson, 1995; Wood, 1991), económico-ambiental (e.g. Russo \& Fouts, 1997) y económico-social-ambiental (e.g. Elkington, 1994, 1997), denotando un aumento en la combinación de enfoques teóricos para el análisis. Un buen ejemplo es la aparición de la TBL (Elkington, 1994, 1997, 2004) y la creación de valor compartido (Porter \& Kramer, 2011, 2006); de hecho, las definiciones contemporáneas de ambos constructos incluyen estos términos (Aguinis \& Glavas, 2012; Ashrafi et al., 2018; Bansal \& Desjardine, 2014). También se encuentra esta congruencia entre las definiciones de RSE (Dahlsrud, 2008) y de SC (Meuer et al., 2020) dado que muchos estudios adoptan una perspectiva amplia incluyendo $-\mathrm{y}$ a veces confundiendo- ambos términos.

En esta época se destaca especialmente la influencia de la teoría de los stakeholders (Donaldson \& Preston, 1995; Freeman, 1984) en el ámbito de la RSE y SC (Clarkson, 1995; Jones, 1995; Moir, 2001; Latapí Agudelo et al., 2019; Linnenluecke \& Griffiths, 2013). El modelo de esta teoría fue tomado por los investigadores preocupados por el vacío pragmático al intentar medir los resultados de la RSE en el campo, por ejemplo el modelo de desempeño social corporativo -DSC- (Lee, 2008). También Carroll (1999) identifica la proliferación de esta teoría e incluye el desarrollo de otros conceptos y teorías que comienzan a ser temas en el ámbito de la RSE. Otro buen ejemplo de esta época es el desarrollo de la pirámide de la RSE de Wood (1991).

También se denota convergencia en relación a para qué o para quién una empresa es responsable o sostenible. Investigadores de ambos campos comienzan a considerar que la empresa es responsable ante una amplia gama de demandas y stakeholders. Los de RSE se centran en las relaciones con los stakeholders, incluyendo las demandas de actores no privilegiados (Freeman, Martin \& Parmar, 2007). Los de SC abogan 
por la integración entre los sistemas económicos-sociales-ambientales y desplazan la atención a la forma en que las empresas pueden sostenerlos.

Por último, en cuanto a las formas de medición del desempeño también hay similitudes. Los instrumentos de medición utilizados para operacionalizar los constructos de RSE y SC (Montiel, 2008; Perdomo \& Escobar, 2011) terminan siendo similares aunque las fuentes de datos sean distintas (Bansal \& Song, 2017), existiendo muchas limitaciones en dichas mediciones (Chatterji, Levine \& Toffel, 2009; Etzion, 2007). Asrafi et al. (2018) destacan que la ambigüedad también se puede observar en los reportes de las empresas que adquieren nombres diferentes refiriéndose a lo mismo.

En la convergencia de ambos constructos se encuentra la dificultad del avance de la investigación de ambos campos separadamente, pero también se visualizan aspectos de complementariedad. Particularmente, el diálogo entre investigadores de ambos enfoques en relación al uso de otras teorías y/o disciplinas como forma de comprensión más acabada del fenómeno.

\section{Confusión: tensiones actuales}

El inicio del nuevo siglo trajo aparejado un nuevo paradigma de tensión en la investigación, que ha dado lugar a la aparición de críticas que constituyen tensiones importantes a tener en cuenta para el avance. La convergencia de 1990 logró confundir los constructos de tal manera que los campos se volvieron difusos (Bansal \& Song, 2017) y algunos autores consideran que ello ha frenado el crecimiento en la investigación (Meuer et al., 2020; Bansal \& Song, 2017). Los investigadores se centraron en la relación de la TBL -el modelo dominante según Mebratu (1998) - con el management estratégico y cómo lograr el equilibrio entre las tres dimensiones, aunque también en las tensiones y críticas. Además, la demanda de conocimiento en estos temas atravesó la agenda académica y se posicionó en agendas empresariales, institucionales y de política pública (Doh \& Guay, 2006; Glavič \& Lukman, 2007; Latapí Agudelo et al., 2019; Perdomo \& Escobar, 2011), abonando aún más a la convergencia.

Una de las primeras tensiones es el excesivo énfasis que se ha dado en RSE al business case -BC-: una mirada instrumental en la que predomina el desempeño financiero en detrimento del social y ambiental y la consecuente necesidad de una mirada más amplia que integre a todos los stakeholders (Carroll \& Shabana, 2010; Hahn \& Figge, 2011). El concepto se asocia al de DSC, sobre el cual se destaca la gran cantidad de publicaciones durante décadas de investigación en management (Linnenluecke \& Griffiths, 2013), siendo una de las más citadas la de Orlitzky, Schmidt, \& Rynes (2003). Los más críticos enfatizan en que las empresas persiguen el desempeño social y ambiental sólo si al hacerlo no se compromete su desempeño financiero (Milne \& Gray, 2013; Slawinski \& Bansal, 2015) y que la RSE se define por intereses comerciales estrechos y puede incluso contribuir a la explotación de los pueblos indígenas (Banerjee, 2008) y a la esclavitud moderna (Crane, 2013), entre otros problemas actuales.

Otra de las tensiones es la falacia de la separación entre la ética y los negocios, la "profecía autocumplida" (Ferraro, Pfeffer \& Sutton, 2005; Freeman et al., 2007), relacionada con que la existencia de argumentos sociales encubiertos por la RSE tiene el riesgo de reproducir gerentes amorales que sólo actúen de manera responsable o sostenible por su propio interés o el de su empresa (Bansal \& Song, 2017). Algunos autores argumentan que estas prácticas están directamente relacionadas con la educación que reciben los gerentes, basada en teorías del management que reproducen una visión reducida del mundo de los negocios (Ghoshal, 2005).

Otros plantean que parte del dilema ético se encuentra en las narrativas tradicionales del capitalismo en cuanto a la dimensión social -trabajo, poder, competencia, ética y dominación- (Freeman et al., 2007) y que el paradigma actual de sustentabilidad dificulta las discusiones sobre el "asalto" del capitalismo al ambiente natural y a las comunidades indígenas, tanto como el poder excesivo de las empresas multinacionales y otras corporaciones (Banerjee, 2011). El propio concepto de stakeholder está en tensión por las nuevas miradas 
sobre los actores organizacionales sin voz ni protagonismo (Freeman et al., 2007), los más débiles sobre los cuales la teoría del management tradicional no se ha preocupado (Banerjee, 2011; Crane, 2013; Sanabria et al., 2015).

También se encuentran en tensión conceptos como la TBL y el DS. El primero por ser una falacia de composición -las partes no necesariamente comprenden el todo- y por no poder contribuir a la SC al no tener en cuenta las tensiones y las compensaciones entre los elementos del sistema (Bansal \& DesJardine, 2014; Ehrenfeld, 2011; Hahn \& Figge, 2011). El segundo por ser demasiado vago, peligroso, elusivo (Mebratu, 1998), por no definir qué necesidades son las que deben satisfacerse, por no decir con qué mecanismos se puede lograr una sociedad ambientalmente sustentable, y por enfocarse en el mantenimiento de una situación para la mejora de las futuras generaciones pero en términos económicos principalmente, teniendo en cuenta el ambiente sólo de manera subsidiaria (Castro, 2004).

Todas estas tensiones nos llevan a pensar que "es posible que [las teorías del management] hayan alentado tácitamente a las organizaciones a comportarse de manera que, en última instancia, destruyan sus sistemas naturales y sociales de sustentación de la vida" (Gladwin et al., 1995, p. 896). Por lo cual, se denota la necesidad de generar nuevos marcos que puedan enfrentarlas y dar respuestas válidas a académicos y empresarios. Parte fundamental de este proceso está en la incorporación de nuevas corrientes de pensamiento heterodoxas al análisis.

\section{Perspectivas de investigación futura}

En primer lugar, y de acuerdo con las diferencias remarcadas entre RSE y SC, existe la posibilidad de profundización de cada uno de los constructos separadamente (Bansal \& Song, 2017). La investigación en RSE puede desarrollar herramientas y mecanismos teóricos que demuestren cómo las organizaciones van cambiando de acuerdo con una perspectiva más amplia de sociedad (Lee, 2008), dado también que la categoría social es una de las menos abordadas (Lockett et al., 2006). O también profundizar en los aspectos normativos y en la responsabilidad moral de los gerentes como teoría normativa de la firma. Esta idea se opone a lo propuesto por Perdomo y Escobar (2011), quienes destacan una evolución progresiva de la investigación en RSE contraria a una de tipo normativa. (Bansal \& Song, 2017; Lockett et al., 2006; Margolis \& Walsh, 2003). En una línea más específica, Aguinis y Glavas (2012) proponen la profundización en los “microfundamentos" de la RSE, basados en acciones e interacciones individuales.

Por su parte, la investigación en SC, puede ahondar en la relación entre los sistemas empresa-sociedad y sus niveles, los conceptos de estabilidad, cambio y resiliencia, y las disrupciones en esos sistemas (Bansal \& Song, 2017; Folke et al., 2002; Linnenluecke \& Griffiths, 2013), o contemplar tensiones y paradojas más allá de la TBL (Hahn, Pinkse et al., 2015). O simplemente profundizar en el constructo SC (e.g. Meuer et al., 2020; Montiel \& Delgado-Ceballos, 2014).

En segundo lugar, en relación con lo analizado sobre la convergencia de ambos constructos y sus complementariedades, la investigación puede incorporar otras teorías y disciplinas que ayuden a comprender la complejidad del fenómeno. Existen ejemplos que relacionan teorías de la Economía, la Administración y otras ciencias sociales con los enfoques de RSE y SC: las teorías de contratos sociales y agencia, de legitimidad, evolucionaria, basada en los recursos, agregada de las entidades, de la firma, del neo-institucionalismo (Lozano et al., 2015; Montiel y Delgado-Ceballos, 2014; Lee, 2008; Aguilera, Rupp et al., 2007; McWilliams et al., 2006; Doh y Guay, 2006; Moir, 2001; Hoffman, 1999). Algunos autores destacan la combinación de más de un enfoque teórico (Aguinis \& Glavas, 2012) y aluden a la integración de otras ciencias sociales al análisis de la RSE para comprender el rol de las organizaciones como actores políticos privados y sus relaciones con los actores políticos públicos (Scherer \& Palazzo, 2011), incluso aplicando este enfoque (Aguilera et al., 2007; Doh \& Guay, 2006). 
También, continuar en la integración de la sustentabilidad como concepto inter y transdisciplinario (Brandt, Ernst et al., 2013; Lang, Wiek et al., 2012), como sistema socioecológico de relaciones (Amato, 2019), dado que va creciendo en los últimos años como objeto de investigación (Bansal \& DesJardine, 2014) pero con escasa difusión en América Latina (Brandt et al., 2013). Los autores sugieren nuevos marcos conceptuales como el sustaincentrism (Gladwin et al., 1995), sustaincentric orientation (Valente, 2012), ecological sustainability (Shrivastava, 1995), corporate sustainable development (Bansal, 2005); los cuales también son concebidos como nuevas corrientes teóricas de la SC (Montiel \& Delgado-Ceballos, 2014) o nuevas áreas de debate (Linnenluecke \& Griffiths, 2013).

En tercer y último lugar, se destaca como perspectiva a futuro la integración de corrientes de pensamiento heterodoxas al análisis de la relación entre empresa-sociedad-ambiente, como los ECA (Adler et al., 2007; Alvesson \& Willmott, 2003; Grey \& Willmott, 2005; Sanabria et al., 2015). Esta perspectiva ayudaría a comprender las tensiones en el campo de RSE y SC, avanzando en las críticas que reciben para generar marcos de análisis más integradores que consideren las tensiones desde distintos puntos de vista: "ideologías, instituciones, intereses e identidades (las cuatro I) que se consideran (a) dominantes, (b) perjudiciales y (c) poco cuestionadas" (Sanabria et al., 2015, p. 211).

\section{Conclusiones}

El análisis de la evolución de la investigación en RSE y SC muestra que cada constructo tuvo su origen en momentos y con objetivos diferentes. La RSE nace a mediados del siglo XX con un claro paradigma relacionado a la moralidad de los empresarios y las empresas, por ello que sus primeras definiciones se asociaron a las obligaciones morales y el deber social. De allí también las raíces teóricas del constructo situadas en la ética.

La SC, en cambio, nace después con un claro sesgo ambiental relacionado con un paradigma sobre el debate "verde" en Administración. Sus primeras definiciones se basaron en la gestión ambiental y el desarrollo económico (una de las causas de asociación de este constructo con el de DS); y sus raíces teóricas en la ciencia, fundamentan su mirada sistémica acerca de la relación empresa-sociedad-ambiente.

Con el avance en la investigación, surge un nuevo paradigma de convergencia entre ambos constructos y las diferencias originarias desaparecieron. Las similitudes en las definiciones y asunciones de la RSE y la SC son evidentes en la literatura analizada. Esta situación ha generado tensiones en el campo de la investigación que reclaman la revisión de los conceptos para lograr una mayor claridad.

Estas tensiones se relacionan con la mirada instrumental de la investigación en RSE en la que ha predominado el desempeño financiero en detrimento del social o ambiental (business case); también se cuestiona que los gerentes pueden estar actuando de manera responsable o sostenible sólo por su propio interés (profecía autocumplida); y se reclama la necesaria integración de todos los stakeholders en el análisis de estos constructos, incluso aquellos invisibilizados. El concepto de la TBL también está en discusión, por no considerar estas tensiones en el análisis y las compensaciones entre los elementos de las dimensiones económicas, sociales y ambientales. En el ámbito de la SC se destacan los reclamos que realizan las corrientes heterodoxas de un cambio de paradigma en los modos de producción y consumo actuales, traducidos en las luchas de poder en contra del capitalismo y el productivismo, principalmente. Tensión que ha sido puesta en escena a nivel mundial por la crisis sanitaria del 2020 asociada a la pandemia del COVID-19.

El paradigma de tensión en la investigación en RSE y SC requiere de perspectivas futuras de investigación para el avance: la profundización de cada constructo separadamente, teniendo en cuenta sus raíces teóricas; la incorporación de otras teorías y disciplinas al análisis, dado el carácter de transdisciplinariedad y complementariedad de los constructos; y la innovación en la incorporación de corrientes teóricas heterodoxas, como los ECA, para integrar todas las tensiones del campo. Avanzar en este paradigma tiene 
el objetivo de dar respuesta a la confusión de las comunidades científicas, los académicos y los empresarios sobre ambos constructos.

Otras líneas de investigación a futuro son el avance empírico en cada constructo, que también es una necesidad que reclama la literatura e incluso la utilización de metodologías cualitativas (Lockett et al., 2006); también sería importante una revisión bibliométrica y cienciométrica complementaria a esta revisión bibliográfica orientada (e.g. Olawumi \& Chan, 2018).

Algunas limitaciones de este trabajo son las propias de una revisión de literatura orientada en donde se han seleccionado artículos, con determinados criterios, sin pretensiones de exhaustividad; y las relacionadas con los problemas de comparabilidad cuando se realizan reviews terciarias (Meuer et al., 2020; Orlitzky et al., 2003). Otra limitante está dada por los aspectos analizados, dado que se realizó un recorte en cuanto a los temas que se tratarían por lo que no se ha ahondado en relación con, por ejemplo, medición de los constructos, o en conceptos que aparecen repetidamente en las búsquedas, como economía circular, transición a la sustentabilidad, entre otros (e.g. Olawumi \& Chan, 2018; Glavič \& Lukman, 2007). Tampoco se ha incorporado la discusión de si las diferencias, similitudes y tensiones también se vislumbran en el campo gerencial, institucional o de política pública, como sucede en el campo científico.

Reconociendo estas limitaciones, el aporte de este trabajo es una mirada sobre la investigación en RSE y SC, ordenando y conectando ideas para comprender cada uno de los términos, definir líneas futuras de acción y dar luz al camino de la investigación. Pensar un nuevo paradigma en donde los investigadores del management puedan reconocer su dominio como comunidad íntegra sobre -y entre- lo social y lo ambiental, además de lo económico, avanzando en las tensiones del campo para dar respuestas a las comunidades científica, académica y empresaria.

\section{Referencias}

Ackerman, R. (1973). How companies respond to social demands. Harvard Business Review, July/August, 88-98.

Acquier, A., Gond, J., \& Pasquero, J. (2011). Rediscovering Howard R. Bowen's legacy the unachieved agenda and continuing relevance of social responsibilities of the businessman. Business \& Society, 50(4), 607-646. https://d oi.org/10.1177/0007650311419251

Adler, P., Forbes, L., \& Willmott, H. (2007). 3 Critical management studies. The Academy of Management Annals, I(1), 119-179. https://doi.org/10.5465/078559808

Aguilera, R., Rupp, D., Williams, C., \& Ganapathi, J. (2007). Putting the S back incorporate social responsibility: A multilevel theory of social change in organizations. Academy of Management Review, 32, 836-863. https://doi .org/10.5465/amr.2007.25275678

Aguinis, H., \& Glavas, A. (2012). What we know and don't know about corporate social responsibility: A review and research agenda. Journal of Management, 38(4), 932-968. https://doi.org/10.1177/0149206311436079

Alvesson, M., \& Willmott, H. (eds.) (2003). Studying management critically. London: Sage

Amato, C. (2019). Revisión bibliográfica sobre sustentabilidad y Ética organizacional: Actores relevantes. Ciencias Administrativas, 7(13), 74-84. https://doi.org/10.24215/23143738e036

Amato, C., Buraschi, M., \& Peretti, M. F. (2016). Orientación de los empresarios de Córdoba-Argentina hacia la sustentabilidad y la responsabilidad social empresarial: identificación de variables asociadas a cada constructo. Revista Contaduria y Administración, 61(1), 84-105. http://dx.doi.org/10.1016/j.cya.2015.10.001

Ashrafi, M., Adams, M., Walker, T., \& Magnan, G. (2018). How corporate social responsibility can be integrated into corporate sustainability: A theoretical review of their relationships. International Journal of Sustainable Development \& World Ecology, 25(8), 672-682. https://doi.org/10.1080/13504509.2018.1471628

Atkinson, G. (2000). Measuring corporate sustainability. Journal of Environmental Planning and Management, 43, 235-252. https://doi.org/10.1080/09640560010694 
Banerjee, S. (2008). Corporate social responsibility: The good, the bad and the ugly. Critical Sociology, 34(1), 51-79. https://doi.org/10.1177/0896920507084623

Banerjee, S. (2011). Critical perspectives on business and natural environment. En P. Bansal \& A. J. Hoffman (Eds.), The Oxford handbook of business and the natural environment. (pp. 272-590) Oxford: Oxford University Press. https://doi.org/10.1093/oxfordhb/9780199584451.003.0031

Bansal, P. (2005). Evolving sustainably: A longitudinal study of corporate sustainable development. Strategic Management Journal, 26(3), 197-218. https://doi.org/10.1002/smj.441

Bansal, P., \& DesJardine, M. (2014). Business sustainability: It is about time. Strategic Organization, 12(1), 70-78. h ttps://doi.org/10.1177/1476127013520265

Bansal, P., \& Song, H. (2017). Similar but not the same: Differentiating corporate sustainability from corporate responsibility. Academy of Management Annals, 11(1), 105-149. https://doi.org/10.5465/annals.2015.0095

Barnard, C. (1938). The functions of the executive. Cambridge: Harvard University Press.

Bowen, H. (1953). Social responsibility of the businessman. New York: Harper.

Brandt, P., Ernst, A., Gralla, F., Luederitz, C., Lang, D., Newig, J., et al. (2013). A review of transdisciplinary research in sustainability science. Ecological economics, 92, 1-15. https://doi.org/10.1016/j.ecolecon.2013.04.008

Carroll, A. (1999). Corporate social responsibility evolution of a definitional construct. Business \& Society, 38(3), 268295. https://doi.org/10.1177/000765039903800303

Carroll, A., \& Shabana, K. (2010). The business case for corporate social responsibility: A review of concepts, research and practice. International Journal of Management Reviews, 12(1), 85-105. https://doi.org/10.1111/j.1468-23 70.2009.00275.x

Castro, C. (2004). Sustainable development: mainstream and critical perspectives. Organization \& Environment, 17(2), 195-225. https://doi.org/10.1177/1086026604264910

Chatterji, A., Levine, D., \& Toffel, M. (2009). How well do social ratings actually measure corporate social responsibility? Journal of Economics \& Management Strategy, 18(1), 125-169. https://doi.org/10.1111/j.1530 $-9134.2009 .00210 . x$

Clarkson, M. (1995). A stakeholder framework for analyzing and evaluating corporate social performance. Academy of Management Review, 20(1), 92-117. https://doi.org/10.5465/amr.1995.9503271994

CMMAyD (1987). Nuestro futuro común (Reporte Brundtland, Vol. 383). Oxford: Oxford Unversity Press.

Crane, A. (2013). Modern slavery as a management practice: Exploring the conditions and capabilities for human exploitation. Academy of Management Review, 38(1), 49-69. https://doi.org/10.5465/amr.2011.0145

Dahlsrud, A. (2008). How corporate social responsibility is defined: an analysis of 37 definitions. Corporate Social Responsibility and Environmental Management, 15(1), 1-13. https://doi.org/10.1002/csr.132

Davis, K. (1973). The case for and against business assumption of Social Responsibilities. Academy of Management Journal, 16, 312-322. https://doi.org/10.5465/255331

Doh, J., \& Guay, T. (2006). Corporate social responsibility, public policy, and NGO activism in Europe and the United States: An institutional- stakeholder perspective. Journal of Management Studies, 43, 47-73. https://doi.org/1 $0.1111 /$ j.1467-6486.2006.00582.x

Donaldson, T., \& Preston, L. (1995). The stakeholder theory of the corporation: Concepts, evidence, and implications. Academy of Management Review, 20(1), 65-91. https://doi.org/10.5465/amr.1995.9503271992

Drucker, P. (1954). The practice of management. New York: Harper \& Row.

Ehrenfeld, R. (2011). Beyond the brave new world: Business for sustainability. In: P. Bansal \& A. J. Hoffman (Eds.), The Oxford handbook of business and the natural environment (pp. 611-619). Oxford: Oxford University Press. https://doi.org/10.1093/oxfordhb/9780199584451.003.0033

Elkington, J. (1994). Towards the sustainable corporation: Win-win-win business strategies for sustainable development. California Management Review, 36(2), 90-100. https://doi.org/10.2307/41165746

Elkington, J. (1997). Cannibals with forks: The triple bottom line of 21st century business. Vancouver: New Society Publishers. https://doi.org/10.5860/choice.36rus-3997 
Elkington, J. (2004). Enter the triple bottom line. In: A. Henriques \& J. Richardson (Eds.), The triple bottom line: Does it all add up? (pp. 1-16). London: EarthScan.

Etzion, D. (2007). Research on organizations and the natural environment, 1992-present: A review. Journal of Management, 33(4), 637-664. https://doi.org/10.1177\%2F0149206307302553

Ferraro, F., Pfeffer, J., \& Sutton, R. (2005). Economics language and assumptions: How theories can become selffulfilling. Academy of Management Review, 30(1), 8-24. https://doi.org/10.5465/amr.2005.15281412

Folke, C., Carpenter, S., Elmqvist, T., Gunderson, L., Holling, C., \& Walker, B. (2002). Resilience and sustainable development: Building adaptive capacity in a world of transformations. AMBIO, 31(5), 437-440. https://doi. org/10.1579/0044-7447-31.5.437

Frederick, W. (1960). The growing concern over social responsibility. California Management Review, 2, pp. 54-61. https://doi.org/10.2307/41165405

Freeman, E. (1984). Strategic Management: A stakeholder approach. Boston: Pitman. https://doi.org/10.1017/cbo97 81139192675.003

Freeman, E., Martin, K., \& Parmar, B. (2007). Stakeholder capitalism. Journal of Business Ethics, 74(4), 303-314. ht tps://doi.org/10.1007/s10551-007-9517-y

Friedman, M. (1970). The social responsibility of business is to increase its profits. New York Times, September 13, 122-126.

Garriga, E., \& Melé, D. (2004). Corporate social responsibility theories: Mapping the territory. Journal of Business Ethics, 53(1-2), 51-71. https://doi.org/10.1023/b:busi.0000039399.90587.34

Gladwin, T., Kennelly, J., \& Krause, T. (1995). Shifting paradigms for sustainable development: Implications for management theory and research. Academy of Management Review, 20(4), 874-907. https://doi.org/10.5465/ amr.1995.9512280024

Glavič, P., \& Lukman, R. (2007). Review of sustainability terms and their definitions. Journal of cleaner production, 15(18), 1875-1885. https://doi.org/10.1016/j.jclepro.2006.12.006

Ghoshal, S. (2005). Bad management theories are destroying good management practices. Academy of Management Learning \& Education, 4(1), 75-91. https://doi.org/10.5465/amle.2005.16132558

Grey, Ch., \& Willmott, H. (2005). Critical management studies: a reader. Oxford: Oxford University Press.

Hahn, T., \& Figge, F. (2011). Beyond the bounded instrumentality in current corporate sustainability research: Toward an inclusive notion of profitability. Journal of Business Ethics, 104(3), 325-345. https://doi.org/10.1007/s105 51-011-0911-0

Hahn, T., Pinkse, J., Preuss, L., \& Figge, F. (2015). Tensions in corporate sustainability: Towards an integrative framework. Journal of Business Ethics, 127(2), 297-316. https://doi.org/10.1007/s10551-014-2047-5

Hart, S. (1995). A natural-resource-based view of the firm. Academy of Management Review, 20(4), 986-1014. https ://doi.org/10.5465/amr.1995.9512280033

Hoffman, A. (1999). Institutional evolution and change: Environmentalism and the US chemical industry. Academy of Management Journal, 42, 351-371. https://doi.org/10.5465/257008

Jones, T. (1995). Instrumental stakeholder theory: A synthesis of ethics and economics. Academy Management Review, 20, 404-437. https://doi.org/10.5465/amr.1995.9507312924

Kuhn, T. (1962). The Structure of Scientific Revolutions. Chicago: University of Chicago Press

Lang, D., Wiek, A., Bergmann, M., Stauffacher, M., Martens, P., Moll, P., et al. (2012). Transdisciplinary research in sustainability science: practice, principles, and challenges. Sustainability Science, 7(1), 25-43. https://doi.org/1 0.1007/s11625-011-0149-x

Latapí Agudelo, M., Jóhannsdóttir, L., \& Davídsdóttir, B. (2019). A literature review of the history and evolution of corporate social responsibility. International Journal of Corporate Social Responsibility, 4(1), 1. https://doi.org/ 10.1186/s40991-018-0039-y 
Lee, M. (2008). A review of the theories of corporate social responsibility: Its evolutionary path and the road ahead. International Journal of Management Reviews, 10(1), 53-73. https://doi.org/10.1111/j.1468-2370.2007.0022 6.x

Lélé, S. (1991). Sustainable development: A critical review. World Development, 19(6), 607-621. https://doi.org/10 $.1016 / 0305-750 x(91) 90197-p$

Linnenluecke, M., \& Griffiths, A. (2013). Firms and sustainability: Mapping the intellectual origins and structure of the corporate sustainability field. Global Environmental Change, 23(1), 382-391. https://doi.org/10.1016/j.g loenvcha.2012.07.007

Lockett, A., Moon, J., \& Visser, W. (2006). Corporate social responsibility in management research: Focus, nature, salience and sources of influence. Journal of Management Studies, 43(1), 115-136. https://doi.org/10.1111/j.1 467-6486.2006.00585.x

Lozano, R. (2008). Envisioning sustainability three-dimensionally. Journal of Cleaner Production, 16, 1838-1846. ht tps://doi.org/10.1016/j.jclepro.2008.02.008

Lozano, R., Carpenter, A., \& Huisingh, D. (2015). A review of 'theories of the firm'and their contributions to Corporate Sustainability. Journal of Cleaner Production, 106, 430-442. https://doi.org/10.1016/j.jclepro.2014 .05 .007

Margolis, J., \& Walsh, J. (2003). Misery loves companies: Rethinking social initiatives by business. Administrative Science Quarterly, 48(2), 268-305. https://doi.org/10.2307/3556659

Marín-Idárraga, D. (2012). Consideraciones epistemológicas en torno al carácter científico de la administración. Innovar, 22(46), 39-52. http://hdl.handle.net/20.500.12010/9644.

Martins, C., \& Pato, M. (2019). Supply chain sustainability: A tertiary literature review. Journal of Cleaner Production, 225, 995-1016. https://doi.org/10.1016/j.jclepro.2019.03.250

McWilliams, A., Siegel, D., \& Wright, P. (2006). Corporate social responsibility: Strategic implications. Journal of Management Studies, 43(1), 1-18. https://doi.org/10.1111/j.1467-6486.2006.00580.x

Meadows, D. H., Meadows, D. L., Randers, J., \& Behrens, W. (1972). Los limites del crecimiento: Informe al Club de Roma sobre el predicamento de la humanidad. México D.F.: Fondo de Cultura Económica.

Mebratu, D. (1998). Sustainability and sustainable development: historical and conceptual review. Environmental impact assessment review, 18(6), 493-520.https://doi.org/10.1016/S0195-9255(98)00019-5

Meuer, J., Koelbel, J., \& Hoffmann, V. (2020). On the nature of corporate sustainability. Organization \& Environment, 33(3), 319-341. https://doi.org/10.1177/1086026619850180

Milne, M., \& Gray, R. (2013). W(h)ither ecology? The triple bottom line, the global reporting initiative, and corporate sustainability reporting. Journal of Business Ethics, 118(1), 13-29. https://doi.org/10.1007/s10551-012-1543-8

Moir, L. (2001). What do we mean by corporate social responsibility? Corporate Governance: The International Journal of Business in Society, 1(2), 16-22. https://doi.org/10.1108/eum0000000005486

Montiel, I. (2008). Corporate social responsibility and corporate sustainability separate pasts, common futures. Organization \& Environment, 21(3), 245-269. https://doi.org/10.1177/1086026608321329

Montiel, I., \& Delgado-Ceballos, J. (2014). Defining and measuring corporate sustainability: Are we there yet? Organization \& Environment, 27(2), 113-139. https://doi.org/10.1177/1086026614526413

Olawumi, T., \& Chan, D. (2018). A scientometric review of global research on sustainability and sustainable development. Journal of cleaner production, 183, 231-250. https://doi.org/10.1016/j.jclepro.2018.02.162

Orlitzky, M., Schmidt, F., \& Rynes, S. (2003). Corporate social and financial performance: A meta-analysis. Organization Studies, 24, 403-441. https://doi.org/10.1177/0170840603024003910

Perdomo, J., \& Escobar, A. (2011). La investigación en RSE: una revisión desde el management. Cuadernos de Administración, 24(43), 193-219. https://doi.org/10.11144/Javeriana.cao24-43.irrd

Porter, M. \& Kramer, M. (2006). Strategy \& society: the link between competitive advantage and corporate social responsibility. Harvard Business Review, 84, 78-92.

Porter, M., \& Kramer, M. (2011). La creación de valor compartido. Harvard Business Review América Latina, 1-18. 
Preston, L., \& Post, J. (1975). Measuring Corporate Responsibility. Journal of General Management, 2(3), 45-52. ht tps://doi.org/10.1177/030630707500200306

Russo, M., \& Fouts, P. (1997). A resource-based perspective on corporate environmental performance and profitability. Academy of Management Journal, 40, 534-559. https://doi.org/10.5465/257052

Sanabria, M., Saavedra, J., \& Smida, A. (2015). Los estudios críticos en administración: origen, evolución y posibilidades de aporte al desarrollo del campo de los estudios organizacionales en América Latina. Revista de la Facultad de Ciencias Económicas: Investigación y Reflexión, 23(1), 209-234. https://doi.org/10.18359/rfce.616

Scherer, A., \& Palazzo, G. (2011). The new political role of business in a globalized world: A review of a new perspective on CSR and its implications for the firm, governance, and democracy. Journal of Management Studies, 48(4), 899-931. https://doi.org/10.1111/j.1467-6486.2010.00950.x

Shrivastava, P. (1995). The role of corporations in achieving ecological sustainability. Academy of Management Review, 20, 936-960. https://doi.org/10.5465/amr.1995.9512280026

Slawinski, N., \& Bansal, P. (2015). Short on time: Intertemporal tensions in business sustainability. Organizaton Science, 26(2), 531-549. https://doi.org/10.1287/orsc.2014.0960

Swanson, D. (1995). Addressing a theoretical problem by reorienting the corporate social performance model. Academy of Management Review, 20, 43-64. https://doi.org/10.5465/amr.1995.9503271990

Valente, M. (2012). Theorizing firm adoption of sustaincentrism. Organization Studies, 33(4), 563-591. https://doi .org/ $10.1177 / 0170840612443455$

Wood, D. (1991). Corporate social performance revisited. Academy of Management Review, 20, 691-718. https://d oi.org/10.5465/amr.1991.4279616 


\section{Anexos}

\section{Anexo A}

TABLA A1, 1

Características de los reviews seleccionados en la etapa 1 de la metodología (ordenados cronológicamente)

\begin{tabular}{|c|c|c|c|c|c|}
\hline Antores & Año & Citas* & Tipo de roviow & $\begin{array}{c}\text { Periodo de } \\
\text { tiempo }\end{array}$ & Tema \\
\hline Lélé & 1991 & 3.204 & Revisión crítica & $\begin{array}{l}\text { No explicita } \\
\text { pero considera } \\
\text { bibliografia } \\
\text { desde } 1970 .\end{array}$ & $\begin{array}{l}\text { Es uno de los primeros autores en analizar el } \\
\text { concepto de DS y sustentabilidad, mostrando la } \\
\text { inconsistencia en la interpretación del término, } \\
\text { mediante una revisión critica de las debilidades del } \\
\text { pensamiento mainstream del DS, tales como la } \\
\text { pobreza y la degradación ambiental. }\end{array}$ \\
\hline Elkington** & $\begin{array}{l}1994 \\
1997 \\
2004\end{array}$ & $\begin{array}{c}3.584 \\
120 \\
539\end{array}$ & $\begin{array}{l}\text { No explicita } \\
\text { pero revisa } \\
\text { literatura. }\end{array}$ & $\begin{array}{l}\text { No explicita } \\
\text { pero considera } \\
\text { bibliografia } \\
\text { desde } 1960 .\end{array}$ & $\begin{array}{l}\text { En su primer trabajo ofrece los cimientos de lo que, } \\
\text { años más tarde, identificariacomo TBL, mostrando } \\
\text { un análisis del cambio de las estrategias } \\
\text { empresariales hacia modelos más "verdes" que } \\
\text { serian las que moverian el siglo XXI. En } 1997 \\
\text { escribe su libro en el que define la TBL y sus tres } \\
\text { dimensiones (económica, social y ambiental), } \\
\text { analizando sus implicancias de cara al nuevo siglo. } \\
\text { En 2004, escribe un capitulo de libro en el que } \\
\text { introduce la TBL resumiendo sus trabajos anteriores } \\
\text { y afianzindose como creador del término. Aqui } \\
\text { identifica los siete mecanismos de la agenda TBL o } \\
\text { revoluciones de la sustentabilidad para los negocios. }\end{array}$ \\
\hline Gladwin et al. & 1995 & 2.819 & $\begin{array}{l}\text { No explicita } \\
\text { pero indica ser } \\
\text { un análisis de } \\
\text { teoriasy } \\
\text { prácticas del } \\
\text { management. }\end{array}$ & $\begin{array}{l}\text { No explicita } \\
\text { pero considera } \\
\text { bibliografia } \\
\text { desde } 1970 .\end{array}$ & $\begin{array}{l}\text { Parte de la existencia de una fractura epistemológica } \\
\text { en las ciencias organizacionales, relacionada con la } \\
\text { disociación entre la humanidad y el entorno natural, } \\
\text { confrontando el impacto de la teoria y las prácticas } \\
\text { del management con la humanidad, el ambiente y la } \\
\text { sustentabilidad. Los autores abogan por una } \\
\text { reintegración y proponen un nuevo paradigma } \\
\text { llamado "sustaincentrism" definiendo sus } \\
\text { implicancias para las organizaciones, la comunidad } \\
\text { y la academia. }\end{array}$ \\
\hline Mebratu & 1998 & 1.668 & $\begin{array}{l}\text { No explicita } \\
\text { pero indica ser } \\
\text { un análisis } \\
\text { sistemático de } \\
\text { definiciones e } \\
\text { interpretaciones } \\
\text { de } \\
\text { sustentabilidad } \\
\text { y DS. }\end{array}$ & $\begin{array}{l}\text { No explicita } \\
\text { pero considera } \\
\text { bibliografia } \\
\text { desde clásicos } \\
\text { del } 1800 .\end{array}$ & $\begin{array}{l}\text { Examina los paralelismos ambientales en la historia } \\
\text { de la humanidad, la evolución del concepto de DS y } \\
\text { las definiciones e interpretaciones actuales. Se } \\
\text { identifican los principales defectos conceptuales de } \\
\text { esas definiciones. Aborda el análisis conceptual } \\
\text { desde tres perspectivas: institucional, ideológicay } \\
\text { académica; y la evolución del concepto en tres } \\
\text { etapas: pre Conferencia de Estocolmo (1972), desde } \\
\text { la Conferencia hasta la CMMAyD (1987), y post } \\
\text { CMMAyD. }\end{array}$ \\
\hline Carroll & 1999 & 10.446 & $\begin{array}{l}\text { Revisión de } \\
\text { definiciones. }\end{array}$ & $1950-1990$ & $\begin{array}{l}\text { Uno de los trabajos más citados en el ámbito de la } \\
\text { RSE; analiza la historia del término identificando su } \\
\text { evolución y los nuevos temas que aparecen en cada } \\
\text { década. Se divide el análisis en: inicios de la RSE } \\
\text { (1950), expansión (1960), proliferación (1970), } \\
\text { inicio de investigaciones empiricas y nuevas } \\
\text { alternativas (1980), y nuevos campos y temas } \\
\text { alternativos (1990). }\end{array}$ \\
\hline Moir & 2001 & 1.412 & $\begin{array}{l}\text { No explicita } \\
\text { pero indica ser } \\
\text { un análisis de } \\
\text { literatura. }\end{array}$ & $\begin{array}{l}\text { No explicita } \\
\text { pero considera } \\
\text { bibliografia } \\
\text { desde } 1960 .\end{array}$ & $\begin{array}{l}\text { Analiza las ideas subyacentes al concepto de RSE } \\
\text { intentando definir cuáles son las responsabilidades } \\
\text { sociales de las empresas (para qué y para quién), } \\
\text { examinando teorias para responder al cómo y al } \\
\text { porqué las empresas pueden emprender acciones de } \\
\text { RSE. }\end{array}$ \\
\hline
\end{tabular}

* Se recurrió a una base general para el número de citas, tomando Google Scholar (https://scholar.google.com) al 28/10/2020.

${ }^{* *}$ En Elkington no se tuvieron en cuenta las citas, dado que es el creador de la TBL y sus trabajos aportan en esta creación.

Fuente: elaboración propia. 
TABLA A1, 2

Características de los reviews seleccionados en la etapa 1 de la metodología (ordenados cronológicamente)

\begin{tabular}{|c|c|c|c|c|c|}
\hline Antores & Año & Citas $^{*}$ & Tipo de reviow & $\begin{array}{c}\text { Periodo de } \\
\text { tiempo }\end{array}$ & Tema \\
\hline $\begin{array}{l}\text { Garriga \& } \\
\text { Melé }\end{array}$ & 2004 & 5.612 & $\begin{array}{l}\text { No explicita } \\
\text { pero indica ser } \\
\text { un analisis de } \\
\text { literatura. }\end{array}$ & $\begin{array}{l}\text { No explicita } \\
\text { pero considera } \\
\text { bibliografia } \\
\text { desde } 1960 .\end{array}$ & $\begin{array}{l}\text { Basindose en que la RSE es un territorio } \\
\text { controversial, complejoy no muy claro, propone } \\
\text { clarificare cel concepto clasificando las teoriasy } \\
\text { enfoques de RSE en cuatro grupos (instrumentales, } \\
\text { politicas, integradoras y éticas). Muestrala } \\
\text { necesidad de una nueva teoria de la relación } \\
\text { empresa-sociedad que tenga en cuenta auatro } \\
\text { dimensiones: rentabilidad, desempento politico, } \\
\text { demandas sociales y valores éticos. }\end{array}$ \\
\hline $\begin{array}{l}\text { McWilliams } \\
\text { et al. }\end{array}$ & 2006 & 3.722 & $\begin{array}{l}\text { No explicita } \\
\text { pero indica ser } \\
\text { un análisis de } \\
\text { literatura. }\end{array}$ & $\begin{array}{l}\text { No explicita } \\
\text { pero considera } \\
\text { bibliografia } \\
\text { desde } 1930 .\end{array}$ & $\begin{array}{l}\text { Es una introduccióna un número especial de la } \\
\text { revista en la cual los autores describen perspectivas } \\
\text { teóricas sobre la RSE, que utilizan para desarrollar } \\
\text { las implicaciones estratégicas de la RSE. Proponen } \\
\text { una agenda para la investigación teórica y empirica. }\end{array}$ \\
\hline $\begin{array}{l}\text { Glavic \& } \\
\text { Lukman }\end{array}$ & 2007 & 1.050 & $\begin{array}{l}\text { No explicita } \\
\text { pero se basa en } \\
\text { definiciones de } \\
\text { DS y } \\
\text { sustentabilidad. }\end{array}$ & $\begin{array}{l}\text { No explicita } \\
\text { pero considera } \\
\text { bibliografia } \\
\text { reciente al } \\
2007 .\end{array}$ & 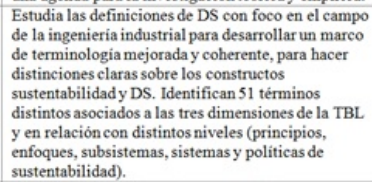 \\
\hline Dahlsrud & 2008 & 5.034 & $\begin{array}{l}\text { Revisión de } \\
\text { literatura de } 37 \\
\text { definiciones de } \\
\text { RSE. }\end{array}$ & $1980-2003$ & $\begin{array}{l}\text { Revisa las definiciones de RSE en la literatura con } \\
\text { el fin de determinar si existe o no confusión en ellas, } \\
\text { concluyendo que eran congruentes y no habia } \\
\text { confusión en ese aspecto, pero si en como la RSE es } \\
\text { construida socialmente en algunos contextos. } \\
\text { Expresa la idea de que una estrategia de RSE debe } \\
\text { estar contextualizada para cada empresa, y lo que es } \\
\text { especifico para una puedo no serlo para otra. }\end{array}$ \\
\hline Lee & 2008 & 1.873 & $\begin{array}{l}\text { No explicita } \\
\text { pero se advierte } \\
\text { un analisis de } \\
\text { literatura. }\end{array}$ & $\begin{array}{l}\text { No explicita } \\
\text { pero considera } \\
\text { bibliografia } \\
\text { desde } 1950 .\end{array}$ & $\begin{array}{l}\text { Analiza la evolución de las teorias sobre RSE } \\
\text { concluyendo que existe una racionalización del } \\
\text { concepto, que implica que a nivel de análisis los } \\
\text { investigadores viraron la discusión sobre el efecto } \\
\text { macro-social al efecto individual-organizacional de } \\
\text { la RSE en las ganancias; y a nivel teórico los } \\
\text { investigadores se estän moviendo de argumentos } \\
\text { ético-normativos explicitos hacia estudios } \\
\text { gerenciales normativos implicitos y orientados al } \\
\text { desempento. }\end{array}$ \\
\hline Montiel & 2008 & 771 & $\begin{array}{l}\text { Revisión de } \\
\text { literatura de } 91 \\
\text { articulos. }\end{array}$ & $1970-2005$ & $\begin{array}{l}\text { Analiza las definiciones de RSE y SC en el tiempo, } \\
\text { revelando diferencias y congruencias entre ambos. } \\
\text { Expone recomendaciones a los investigadores } \\
\text { interesados en "integrar" ambos terminos. }\end{array}$ \\
\hline Lozano & 2008 & 729 & $\begin{array}{l}\text { No explicita } \\
\text { pero se basa en } \\
\text { reviews sobre } \\
\text { representaciones } \\
\text { visuales para } \\
\text { comprender la } \\
\text { sustentabilidad. }\end{array}$ & $\begin{array}{l}\text { No explicita } \\
\text { pero considera } \\
\text { bibliografia } \\
\text { reciente al } \\
2008 .\end{array}$ & $\begin{array}{l}\text { Propone un modelo ode representación del concepto } \\
\text { de sustentabilidad más holistico denominado Two } \\
\text { Tlered Sustainability Equilibriay representa las tres } \\
\text { dimensiones se la sustentabilidad conjuntamente } \\
\text { con las perspectivas de corto, mediano y largo } \\
\text { plazo. Integra las debilidades que otros modelos } \\
\text { posenen en cuanto a que todos ellos son altamente } \\
\text { antropocéntricos, compartimentados y carentes de } \\
\text { completitudy continuidad. }\end{array}$ \\
\hline
\end{tabular}

TABLA A1, 3

Características de los reviews seleccionados en la etapa 1 de la metodología (ordenados cronológicamente)

\begin{tabular}{|c|c|c|c|c|c|}
\hline Autores & Año & Citas* & Tipo de review & $\begin{array}{l}\text { Periodo de } \\
\text { tiempo }\end{array}$ & Tema \\
\hline $\begin{array}{l}\text { Carroll \& } \\
\text { Shabana }\end{array}$ & 2010 & 4.118 & $\begin{array}{l}\text { No explicita } \\
\text { pero indica ser } \\
\text { un análisis de } \\
\text { literatura. }\end{array}$ & $\begin{array}{l}\text { No explicita } \\
\text { pero considera } \\
\text { bibliografia } \\
\text { desde } 1950 .\end{array}$ & $\begin{array}{l}\text { Describe y caracteriza el "business case" -BC- } \\
\text { para la RSE, es decir, los argumentos o } \\
\text { justificaciones que apoyan o documentan por } \\
\text { qué la comunidad empresarial debe aceptar y } \\
\text { promover la "causa" de la RSE. Destaca una } \\
\text { visión reducida del BC que justifica las } \\
\text { actividades de RSE sólo cuando produce } \\
\text { directamente mejoras en el desempeño } \\
\text { financiero; y que una mirada más amplia es } \\
\text { necesaria. }\end{array}$ \\
\hline $\begin{array}{l}\text { Scherer \& } \\
\text { Palazzo }\end{array}$ & 2011 & 1.895 & $\begin{array}{l}\text { Revisión de } \\
\text { literatura. }\end{array}$ & $\begin{array}{l}\text { No explicita } \\
\text { pero considera } \\
\text { bibliografia } \\
\text { desde } 1970 .\end{array}$ & $\begin{array}{l}\text { Parte del supuesto que la globalización ha } \\
\text { generado nuevos roles en las empresas los } \\
\text { cuales, muchas veces, eran roles asignados al } \\
\text { gobiemo. Analiza la literatura tomando a las } \\
\text { empresas como actores politicos, con } \\
\text { responsabilidades sociales y politicas, pensando } \\
\text { en la politizaciónde la RSE (vista desde } \\
\text { aspectos de la gobernanza y la democracia). }\end{array}$ \\
\hline $\begin{array}{l}\text { Aguinis \& } \\
\text { Glavas }\end{array}$ & 2012 & 3.000 & $\begin{array}{l}\text { Estudio } \\
\text { bibliométrico. }\end{array}$ & 1970-2011 & $\begin{array}{l}\text { Identifica aspectos de la RSE -predictores, } \\
\text { salidas, medidores y moderadores-y sus } \\
\text { relaciones en tres niveles de analisis: } \\
\text { institucional, organizacional e individual. } \\
\text { Provee un marco conceptual que integra todas } \\
\text { estas variables. }\end{array}$ \\
\hline Lang et al. & 2012 & 1.742 & $\begin{array}{l}\text { No explicita } \\
\text { pero se advierte } \\
\text { un análisis de } \\
\text { literatura. }\end{array}$ & $\begin{array}{l}\text { No explicita } \\
\text { pero considera } \\
\text { bibliografia } \\
\text { desde } 1990 .\end{array}$ & $\begin{array}{l}\text { Sintetiza y estructura una serie de principios en } \\
\text { relación con la transidisciplinaridad del concepto } \\
\text { de sustentabilidad, en función de que los } \\
\text { desafios del propio concepto exigen nuevas } \\
\text { formas de conocimiento en cuanto a producción } \\
\text { y toma de decisiones. }\end{array}$ \\
\hline Brandt tet al. & 2013 & 563 & $\begin{array}{l}\text { Revisión } \\
\text { bibliométrica. }\end{array}$ & $1970-2011$ & $\begin{array}{l}\text { Identifica los temas centrales relacionados con } \\
\text { la investigación transdisciplinaria en } \\
\text { sustentabilidad,d de acuerdo con tres } \\
\text { caracteristicas: fases del proceso, tipos de } \\
\text { conocimiento que se crean y grado de } \\
\text { participación de los profesionales. Destaca la } \\
\text { falta de uso de terminologia comunn entre las } \\
\text { comunidades cientificas y el desarrollo de } \\
\text { métodos apropiados. }\end{array}$ \\
\hline $\begin{array}{l}\text { Montiel \& } \\
\text { Delgado- } \\
\text { Ceballos }\end{array}$ & 2014 & 378 & $\begin{array}{l}\text { Revisión de } \\
\text { literatura. }\end{array}$ & $1995-2013$ & $\begin{array}{l}\text { Analiza literatura sobre SC, identifica } \\
\text { definiciones, teerias y mediciones. Los } \\
\text { resultados muestran que hay diferentes enfoques } \\
\text { para definir, teorizar y medir el constructo, y } \\
\text { eso depende en parte del target académico o } \\
\text { profesional. }\end{array}$ \\
\hline $\begin{array}{l}\text { Bansal \& } \\
\text { DesJardine }\end{array}$ & 2014 & 403 & $\begin{array}{l}\text { No explicita } \\
\text { pero se advierte } \\
\text { análisis de } \\
\text { literatura. }\end{array}$ & $\begin{array}{l}\text { No explicita } \\
\text { pero considera } \\
\text { bibliografia } \\
\text { desde } 1970 .\end{array}$ & $\begin{array}{l}\text { Profundiza en los conceptos de RSE y SC } \\
\text { destacando que el tiempo es central en el } \\
\text { análisis de la SC, diferenciándola asi de la RSE. } \\
\text { La SC se relaciona con decisiones estratégicas } \\
\text { de corto y largo plazo, mientras que para la RSE } \\
\text { las decisiones son cortoplacista. }\end{array}$ \\
\hline
\end{tabular}


TABLA A1, 4

Características de los reviews seleccionados en la etapa 1 de la metodología (ordenados cronológicamente)

\begin{tabular}{|c|c|c|c|c|c|}
\hline Autores & Ano & Citas ${ }^{\star}$ & Tipo de review & $\begin{array}{c}\text { Periodo de } \\
\text { tiempo }\end{array}$ & Tema \\
\hline Lozano et al. & 2015 & 205 & $\begin{array}{l}\text { No explicita } \\
\text { pero se advierte } \\
\text { análisis de } \\
\text { literatura. }\end{array}$ & $\begin{array}{l}\text { No explicita } \\
\text { pero considera } \\
\text { bibliografia } \\
\text { desde } \\
\text { principios de } \\
\text { siglo XX. }\end{array}$ & $\begin{array}{l}\text { Presenta las teorias de la firma más utilizadas y se } \\
\text { analizan sus contribuciones a la sustentabilidad a } \\
\text { través de una perspectiva interpretativa. Propone una } \\
\text { teoria integral, "teoria de la firma orientada a la } \\
\text { sustentabilidad", que se basa en elementos de las } \\
\text { teorias analizadas pero de manera relacional y } \\
\text { complementaria. }\end{array}$ \\
\hline Hahn et al. & 2015 & 494 & $\begin{array}{l}\text { No explicita } \\
\text { pero se advierte } \\
\text { análisis de } \\
\text { literatura. }\end{array}$ & $\begin{array}{l}\text { No explicita } \\
\text { pero considera } \\
\text { bibliografia } \\
\text { desde } 1990 .\end{array}$ & $\begin{array}{l}\text { Analiza las tensiones que se dan en cuanto al término } \\
\text { SC, dada la integración simultánea de variables } \\
\text { económicas, sociales y ambientales. Propone un } \\
\text { marco conceptual que integra las dimensiones de } \\
\text { tiempo y espacio en diferentes niveles (sistémico, } \\
\text { organizacional e individual), por sobre la tradicional } \\
\text { triada de la TBL. }\end{array}$ \\
\hline $\begin{array}{l}\text { Bansal \& } \\
\text { Song }\end{array}$ & 2017 & 237 & $\begin{array}{l}\text { No explicita } \\
\text { pero indica ser } \\
\text { un análisis de } \\
\text { literatura. }\end{array}$ & $\begin{array}{l}\text { No explicita } \\
\text { pero considera } \\
\text { articulos en la } \\
\text { bibliografia } \\
\text { desde } 1930 .\end{array}$ & $\begin{array}{l}\text { Analiza el desarrollo que ha tenido cada uno los } \\
\text { constructos RSE y SC en la literatura, argumentando } \\
\text { sus diferencias, similitudes y el actual campo borroso } \\
\text { entre ambas. Propone una agenda que contemple la } \\
\text { continuidad de la investigación de cada uno por } \\
\text { separado, teniendo en cuenta sus raices teóricas y } \\
\text { tradiciones pero en el marco de sistemas económicos } \\
\text { y sociales más resilientes. }\end{array}$ \\
\hline Ashrafi et al. & 2018 & 38 & $\begin{array}{l}\text { No explicita } \\
\text { pero indica ser } \\
\text { una revisión } \\
\text { teórica. }\end{array}$ & $\begin{array}{l}\text { No explicita } \\
\text { pero considera } \\
\text { bibliografia } \\
\text { desde } 1950 .\end{array}$ & $\begin{array}{l}\text { Analiza los conceptos de RSE y SC con el objetivo } \\
\text { de mostrar sus diferencias, reconociendo la } \\
\text { convergencia de los mismos en los últimos tiempos. } \\
\text { Desarrolla una representación gráfica de la } \\
\text { interrelación de ambos, incluyendo el concepto de } \\
\text { DS, con el objetivo de mejorar la comprensión de } \\
\text { cómo ambos conceptos pueden integrarse. }\end{array}$ \\
\hline $\begin{array}{l}\text { Olawumi \& } \\
\text { Chan }\end{array}$ & 2018 & 180 & $\begin{array}{l}\text { Revisión } \\
\text { cienciométrica. }\end{array}$ & 1991-2016 & $\begin{array}{l}\text { Realiza un análisis cienciométrico de publicaciones } \\
\text { relacionadas con sustentabilidad y DS. Muestra } \\
\text { resultados sobre la evolución de los términos, en } \\
\text { relación a las citas, trabajos más citados, autores y co } \\
\text { autores más relevantes, journals y paises más } \\
\text { importantes. También hay un análisis de clusters que } \\
\text { muestra los términos asociados a estos constructos } \\
\text { que más han sido utilizados. El paper tiene una } \\
\text { orientación a la "construcción sostenible" por lo que } \\
\text { también destacan especificidades de esta temática. }\end{array}$ \\
\hline Latapi et al. & 2019 & 70 & $\begin{array}{l}\text { Revisión } \\
\text { sistemática. }\end{array}$ & $1930-2018$ & $\begin{array}{l}\text { Desarrolla la evolución de la RSE como paradigma } \\
\text { conceptual mediante revisión bibliográfica sobre las } \\
\text { contribuciones académicas al concepto, así como } \\
\text { otros eventos importantes que han conformado su } \\
\text { comprensión y definición. Divide el análisis en } \\
\text { décadas y se muestran lineas de tiempo con las } \\
\text { contribuciones más importantes. }\end{array}$ \\
\hline Meuer et al. & 2020 & 11 & $\begin{array}{l}\text { Revisión } \\
\text { sistemática } \\
\text { sobre } 33 \\
\text { definiciones de } \\
\text { SC. }\end{array}$ & $1983-2018$ & $\begin{array}{l}\text { Compara y contrasta } 33 \text { definiciones seminales sobre } \\
\text { SC, diferenciando sus atributos principales, desde } \\
\text { una perspectiva aristotélica. Presenta los resultados } \\
\text { de manera gráfica en lo que llama El Cubo de la SC, } \\
\text { en donde se destacan los aspectos esenciales del } \\
\text { término, de acuerdo con lo analizado. }\end{array}$ \\
\hline
\end{tabular}

\section{Notas}

* Artículo de investigación.

1 "La preocupación motivadora de los [ECA] no son ni los fracasos personales de los administradores individuales ni la mala gestión de organizaciones específicas, sino la injusticia social y la destructividad ambiental de los sistemas sociales y económicos más amplios a los que estos administradores y organizaciones sirven y reproducen” (Adler, Forbes \& Willmott, 2007, p. 2).

2 Se considera paradigma a un patrón de investigación aceptado por una comunidad científica, que tiene vigencia por un determinado tiempo y a partir del cual se explica una realidad (Kuhn, 1962), dejando de lado el debate acerca de que en las ciencias sociales y administrativas no se puede hablar de cambios de paradigma desde la perspectiva kuhniana, sino más bien de discusiones paradigmáticas (Marín-Idárraga, 2012).

$3 \mathrm{Al}$ momento de la corrección de este trabajo se estaba transitando la crisis sanitaria global COVID-19 por lo que se puede afianzar la idea de un quinto paradigma, dado que la pandemia reveló cuestiones asociadas a la RSE y a la SC.

4 Mebratu (1998) aclara que no fue la CMMAyD quien acuñó el término DS, como muchos afirman, sino que fue incluido por primera vez en 1980 en la Estrategia Mundial de Conservación.

\section{Licencia Creative Commons CC BY 4.0}

Para citar este artículo: Amato, C. N. (2021). Investigación en Responsabilidad Social Empresaria y Sustentabilidad Corporativa: evolución, tensiones actuales y perspectivas futuras. Cuadernos de Administración, 34. https://doi.org/10.11144/Javeriana.cao34.irsesc 